\title{
Investigations on the Relativistic Interactions in One- Electron Atoms with Modified Anharmonic Oscillator
}

\section{Abstract}

The bound-state solutions of the modified Dirac equation (m.d.e.) for the modified anharmonic oscillator (m.a.o.) are presented exactly for arbitrary spin-orbit quantum number $k(\tilde{k})$ by means Bopp's shift method instead to solving (m.d.e.) with star product, in the framework of noncommutativity three dimensional real space (NC: 3D-RS). The exact corrections for $n^{\text {th }}$ excited states are found straightforwardly for interactions in one-electron atoms by applying the standard perturbation theory. Furthermore, the obtained corrections of energies are depended on two infinitesimal parameters $\left(\Theta_{i j}, \chi_{i j}\right) \equiv \varepsilon_{i j}^{k}\left(\Theta_{k}, \chi_{k}\right)$ which induced by position-position noncommutativity, in addition to the non-relativistic quantum mechanics $(n, j, l=l \pm 1 / 2, m)$ and $(n, j=\tilde{l} \pm \tilde{s}, \tilde{m})$ under spin-symmetry and p-spin symmetry in (NC: 3D-RS), respectively. In limit of parameters $\left(\Theta_{k}, \chi_{k}\right) \rightarrow(0,0)$, the energy equation is consistent with the results of ordinary relativistic quantum mechanics.

Keywords: Anharmonic oscillator; Noncommutative space; Star product; Bopp's shift method; Dirac equation

Abbreviations: MOA: Modified Anharmonic Oscillator; (NC: 3D-RS): Noncommutativity Three Dimensional Real Space; MDE: Modified Dirac Equation; (NCCRs): NC Canonical Commutations Relations

\section{Introduction}

One of the interesting problems of the relativistic quantum mechanics is to find exact solutions to the Klein-Gordon (to the treatment of a zero-spin particle) and Dirac (spin $1 / 2$ particles and anti-particles) equations for certain potentials of the physical interest, in recent years, considerable efforts have been done to obtain the analytical solution of central and non-central physics problems for different areas of atoms, nuclei, and hadrons, numerous papers of the physicist have discussed in details all the necessary information for the quantum system and in particularly the bound states solutions [1-21]. Some of these potentials are known to play important roles in many fields, one of such potential is the anharmonic oscillator has been a subject of many studies, it is a central potential of nuclear shell model, etc $[20,21]$. The ordinary quantum structures obey the standard Weyl-Heisenberg algebra in both Schrödinger and Heisenberg (the operators are depended on time) pictures, respectively, as (Throughout this paper the natural unit $c=\hbar=1$ are employed):

$$
\begin{aligned}
& {\left[x_{i}, p_{j}\right]=\left[x_{i}(t), p_{j}(t)\right]=i \delta_{i j}} \\
& {\left[x_{i}, x_{j}\right]=\left[p_{i}, p_{j}\right]=\left[x_{i}(t), x_{j}(t)\right]=\left[p_{i}(t), p_{j}(t)\right]=0}
\end{aligned}
$$

where the two operators $\left(x_{i}(t), p_{i}(t)\right)$ in Heisenberg picture are related to the corresponding operators $\left(x_{i}, p_{i}\right)$ in Schrödinger picture from the following projections relations:

$$
\begin{aligned}
& x_{i}(t)=\exp \left(i \hat{H}\left(t-t_{0}\right)\right) x_{i} \exp \left(-i \hat{H}\left(t-t_{0}\right)\right) \\
& p_{i}(t)=\exp \left(i \hat{H}\left(t-t_{0}\right)\right) p_{i} \exp \left(-i \hat{H}\left(t-t_{0}\right)\right)
\end{aligned}
$$

here $\hat{H}$ denote to the ordinary quantum Hamiltonian operator. In addition, for spin $1 / 2$ particles described by the Dirac equation, experiment tells us that must satisfy Fermi Dirac statistics obey the restriction of Pauli, which imply to gives the only non-null equal-time anti-commutator for field operators as follows:

$$
\bar{\Psi}_{\beta}(t, \mathrm{r})=\Psi^{+}{ }_{\beta}(t, \mathrm{r}) \gamma^{0}
$$

with $\bar{\Psi}_{\beta}(t, \mathrm{r})=\Psi^{+}{ }_{\beta}(t, \mathrm{r}) \gamma^{0}$. Very recently, many authors have worked on solving these equations with physical potential in the new structure of quantum mechanics, known by $\mathrm{NC}$ quantum mechanics, which known firstly H Snyder [22], to obtaining profound and new applications for different areas of matter sciences in the microscopic and nano scales [23-68]. It is important to noticing that, the new quantum structure of NC space based on the following NC canonical commutations relations (NCCRs) in both Schrödinger and Heisenberg pictures, respectively, as follows [23-60]:

$$
\begin{aligned}
& {\left[\begin{array}{c}
* \\
\hat{x}_{i}, \hat{p}_{j}
\end{array}\right]=\left[\hat{x}_{i}(t)^{*}, \hat{p}_{j}(t)\right]=i \delta_{i j},\left[\begin{array}{c}
* \\
\hat{x}_{i}, \hat{x}_{j}
\end{array}\right]=\left[\hat{x}_{i}(t)^{*}, \hat{x}_{j}(t)\right]=i \theta_{i j}} \\
& {\left[\hat{p}_{i}^{*}, \hat{p}_{j}\right]=\left[\hat{p}_{i}(t), \hat{p}_{j}(t)\right]=0}
\end{aligned}
$$


Where the two new operators $\left(\hat{x}_{i}(t), \hat{p}_{i}(t)\right)$ in Heisenberg picture are related to the corresponding new operators $\left(\hat{x}_{i}, \hat{p}_{i}\right)$ in Schrödinger picture from the new projections relations:

$$
\begin{aligned}
& \hat{x}_{i}(t)=\exp \left(i \hat{H}_{n c}\left(t-t_{0}\right)\right) * \hat{x}_{i} * \exp \left(-i \hat{H}_{n c}\left(t-t_{0}\right)\right) \\
& \hat{p}_{i}(t)=\exp \left(i \hat{H}_{n c}\left(t-t_{0}\right)\right) * \hat{p}_{i} * \exp \left(-i \hat{H}_{n c}\left(t-t_{0}\right)\right)
\end{aligned}
$$

with $\hat{H}_{n c}$ being the Hamiltonian operator of the quantum system described on (NC: 3D-RS) symmetries. The very small parameters $\theta^{\mu \nu} \quad$ (compared to the energy) are elements of anti symmetric real matrix of dimension $\frac{(\text { length })^{2}}{{ }^{2}}$ and $(*)$ denote to the new star product (the Moyal-Weyl product), which is generalized between two arbitrary functions $f(x) \rightarrow \hat{f}(\hat{x})$ and $g(x) \rightarrow \hat{g}(\hat{x})$ to $\hat{f}(\hat{x}) \hat{g}(\hat{x}) \equiv(f * g)(x)$ instead of the usual product $(f g)(x)$ in ordinary three dimensional spaces [23-68]:

$$
\hat{f}(\hat{x}) \hat{g}(\hat{x}) \equiv(f * g)(x) \equiv \exp \left(\frac { i } { 2 } \theta ^ { \mu v } \partial _ { \mu \nu } ^ { x } \partial _ { \nu } ^ { x } ( f g ) ( x , p ) \equiv \left(f g-\left.\frac{i}{2} \theta^{\mu v} \partial_{\mu}^{x} f \partial_{v}^{x} g\right|_{\left(x^{\mu}=x^{\nu v}\right)}+O\left(\theta^{2}\right)\right.\right.
$$

where $\hat{f}(\hat{x})$ and $\hat{g}(\hat{x})$ are the new function in (NC: 3D-

RS), the following term $\left(-\frac{i}{2} \theta^{m n} \partial_{\mu}^{x} f(x) \partial_{\nu}^{x} g(x)\right)$ is induced by (space-space) noncommutativity properties and $o\left(\theta^{2}\right)$ stands for the second and higher order terms of $\theta$, a Bopp's shift method can be used, instead of solving any quantum systems by using directly star product procedure [23-55]:

$$
\left[\hat{x}_{i}, \hat{x}_{j}\right]=\left[\hat{x}_{i}(t), \hat{x}_{j}(t)\right]=i \theta_{i j} \text { and }\left[\hat{p}_{i}, \hat{p}_{j}\right]=\left[\hat{p}_{i}(t), \hat{p}_{j}(t)\right]=0
$$

The three-generalized coordinates $\left(\hat{x}=\hat{x}_{1}, \hat{y}=\hat{x}_{2}, \hat{z}=\hat{x}_{3}\right)$ in the NC space are depended with corresponding three-usual generalized positions $(x, y, z)$ and momentum coordinates $\left(p_{x}, p_{y}, p_{z}\right)$ by the following relations, as follows [25,28,29,32-34,37-47]:

$$
\begin{aligned}
& \hat{x}=x-\frac{\theta_{12}}{2} p_{y}-\frac{\theta_{13}}{2} p_{z}, \quad \hat{y}=y-\frac{\theta_{21}}{2} p_{x}-\frac{\theta_{23}}{2} p_{z} \\
& \hat{z}=z-\frac{\theta_{31}}{2} p_{x}-\frac{\theta_{32}}{2} p_{y}
\end{aligned}
$$

The non-vanish-commutators in (NC-3D: RS) can be determined as follows:

$$
\begin{aligned}
& {\left[\hat{x}, \hat{p}_{x}\right]=\left[\hat{y}, \hat{p}_{y}\right]=\left[\hat{z}, \hat{p}_{z}\right]=i,} \\
& {[\hat{x}, \hat{y}]=i \theta_{12},[\hat{x}, \hat{z}]=i \theta_{13},[\hat{y}, \hat{z}]=i \theta_{23}}
\end{aligned}
$$

which allow us to getting the operator $\hat{r}^{2}$ on NC three dimensional spaces as follows [25,28,29,32,33,34,37-48]:

$$
\hat{r}^{2}=r^{2}-\overrightarrow{\mathbf{L}} \vec{\Theta}
$$

Where the coupling $\mathbf{L} \Theta$ is given by $\left(\Theta_{i j}=\theta_{i j} / 2\right)$ :

$$
\mathbf{L} \Theta \equiv L_{x} \Theta_{12}+L_{y} \Theta_{23}+L_{z} \Theta_{13}
$$

with $L_{y}=\mathrm{zp}_{\mathrm{x}}-\mathrm{xp}_{\mathrm{z}}, L_{y}=\mathrm{zp}_{\mathrm{x}}-\mathrm{xp}_{\mathrm{z}}$ and $L_{z}=x p_{y}-y p_{x}$. Furthermore, the new equal-time anti-commutator for fermionic field operators' noncommutative spaces can be expressed in the following postulate relations:

$$
\begin{aligned}
& \left\{\hat{\Psi}_{\alpha}(t, \mathrm{r}), \hat{\bar{\Psi}}_{\beta}\left(t, \mathrm{r}^{\prime}\right)\right\}=i\left(\gamma^{0}\right)_{\alpha \beta} \delta^{3}\left(\mathrm{r}-\mathrm{r}^{\prime}\right) \\
& \left\{\hat{\Psi}_{\alpha}(t, \mathrm{r}), \hat{\Psi}_{\alpha}\left(t, \mathrm{r}^{\prime}\right)\right\}=\left\{\hat{\bar{\Psi}}_{\alpha}(t, \mathrm{r}),{ }^{*} \hat{\bar{\Psi}}_{\beta}\left(t, \mathrm{r}^{\prime}\right)\right\}=i \theta_{\alpha \beta} \delta^{3}\left(\mathrm{r}-\mathrm{r}^{\prime}\right)
\end{aligned}
$$

Here $\mathrm{T}$ is the time-ordered product. The purpose of the present work is to extend and present the solution of the Dirac equation with spin-1/2 particle moving in (m.a.o.) potential of the new form:

$$
V_{a o}(\hat{r})=\frac{1}{2} M \omega^{2} r^{2}+\frac{\alpha}{2 M r^{2}}+\left\{\begin{array}{l}
\left(\frac{\alpha}{2 M r^{4}}-\frac{1}{2} M \omega^{2}\right) \overrightarrow{\mathbf{L}} \vec{\Theta} \text { for the spin symmetric case } \\
\left(\frac{\alpha}{2 M r^{4}}-\frac{1}{2} M \omega^{2}\right) \tilde{\mathbf{L}} \vec{\Theta} \text { for the p-spin symmetric case }
\end{array}\right.
$$

In (NC: 3D-RS) using the generalization Bopp's shift method to discover the new symmetries and a possibility to obtain another applications to this potential in different fields. This work based essentially on our previously works [23-48]. The outline of our recently article is as follows: In next section, we briefly review the Dirac equation with anharmonic oscillator on based to [1821]. In section three, we give a description of the Bopp's shift method for the (m.d.e.) with (m.a.o).Then in section four, we apply standard perturbation theory to establish exact modifications at

first order of infinitesimal parameters $(\Theta, \chi)$ for the perturbed Dirac equation in (NC-3D: RS) for spin-orbital (pseudo-spin orbital) and the relativistic magnetic spectrum for (m.a.o.). In the fifth section, we resume the global spectrum and corresponding NC Hamiltonian for (m.a.o.). Finally, some important concluding remarks are drawn from the present study in last section.

\section{Review the Dirac equation for anharmonic oscillator in ordinary quantum}

We start this section by considering a relativistic particle in spherically symmetric for the potential $V(r, \theta)$ which known by anharmonic oscillator, given by in the main reference [21]:

$$
V(r, \theta)=\frac{1}{2} M \omega^{2} r^{2}+\frac{\alpha}{2 M r^{2}}+\frac{\eta}{2 M r^{2} \sin (\theta)} \stackrel{\eta \rightarrow 0}{\longrightarrow} V(r)=\frac{1}{2} M \omega^{2} r^{2}+\frac{\alpha}{2 M r^{2}}
$$


where $M, \omega,(\alpha$ and $\eta$ )denote the rest mass, frequency of particle and dimensionless parameters. The Dirac equation describing a fermionic particle (spin-1/2 particle) with scalar $S(r, \theta)$ and vector $V(r, \theta)$ potentials is given by [18-21]:

$$
(\alpha \mathrm{P}+\beta(M+S(r, \theta))) \Psi(r, \theta, \phi)=(E-V(r, \theta)) \Psi(r, \theta, \phi)
$$

here $M$ are $E$ the fermions' mass and the relativistic energy while $\left(\alpha_{i}=\left(\begin{array}{cc}0 & \sigma_{i} \\ \sigma_{i} & 0\end{array}\right), \beta=\left(\begin{array}{cc}I_{2 \times 2} & 0 \\ 0 & I_{2 \times 2}\end{array}\right)\right)$ are the usual Dirac matrices, the spinor $\Psi(r, \theta, \varphi)$ can be expressed as [21]:

$$
\Psi_{n k}(r, \theta, \phi)=\left(\begin{array}{c}
f_{n k}(\vec{r}) \\
g_{n k}(\vec{r})
\end{array}\right)=\frac{1}{r}\left(\begin{array}{c}
F_{n k}(r) Y_{j m}^{l}(\theta, \phi) \\
i G_{n \tilde{k}}(r) Y_{j m}^{l}(\theta, \phi)
\end{array}\right)
$$

where $\sigma_{1}=\left(\begin{array}{ll}0 & 1 \\ 1 & 0\end{array}\right), \sigma_{2}=\left(\begin{array}{cc}0 & -i \\ i & 0\end{array}\right)$ and $\sigma_{3}=\left(\begin{array}{cc}1 & 0 \\ 0 & -1\end{array}\right)$ and are $2 \times 2$ three Pauli matrices while $k(\tilde{k})$ is related to the total angular momentum quantum numbers for spin symmetry $l$ and p-spin symmetry $\tilde{l}$ as [18-21]:

$$
k=\left\{\begin{array}{l}
-(l+1) \text { if }-(\mathrm{j}+1 / 2),\left(\mathrm{s}_{1 / 2}, p_{3 / 2}, e t c\right), j=l+\frac{1}{2}, \text { aligned } \operatorname{spin}(\mathrm{k}<0) \\
+l \quad \text { if } j=l+\frac{1}{2},\left(\mathrm{p}_{1 / 2}, d_{3 / 2}, \text { etc }\right), j=l-\frac{1}{2}, \text { unaligned } \operatorname{spin}(\mathrm{k}>0)
\end{array}\right.
$$

and

$\tilde{k}=\left\{\begin{array}{l}-\tilde{l} \quad \text { if }-(\mathrm{j}+1 / 2),\left(\mathrm{s}_{1 / 2}, p_{3 / 2}, \text { etc }\right), j=\tilde{l}-\frac{1}{2}, \text { aligned } \operatorname{spin}(\mathrm{k}\langle 0) \\ +(\tilde{l}+1) \quad \text { if } j=\tilde{l}+\frac{1}{2},\left(\mathrm{p}_{1 / 2}, d_{3 / 2}, \text { etc }\right), j=\tilde{l}+\frac{1}{2}, \text { unaligned } \operatorname{spin}(\mathrm{k}>0)\end{array}\right.$

The radial functions $\left(F_{n k}(r), G_{n k}(r)\right)$ are obtained by solving the following differential equations [18-21]:

$$
\left[\frac{d^{2}}{d r^{2}}-\frac{k(k+1)}{r^{2}}-\left(M+E_{n k}-\Delta(r)\left(M-E_{n k}+\Sigma(r)\right)+\frac{\left.\frac{d \Delta(r)}{d r}\left(\frac{d}{d r}+\frac{k}{r}\right)\right)}{M-E_{n k}+\Sigma(r)}\right] F_{n k}(r)=0\right.
$$

and

$$
\left[\frac{d^{2}}{d r^{2}}-\frac{k(k-1)}{r^{2}}\left(M+E_{n k}-\Delta(r)\left(M-E_{n k}+\Sigma(r)\right)+\frac{\frac{d \Sigma(r)}{d r}\left(\frac{d}{d r}+\frac{k}{r}\right)}{M+E_{n k}-\Delta(r)}\right)\right] G_{n \tilde{k}}(r)=0
$$

The exact spin symmetry corresponding $\frac{d \Delta(r)}{d r}=0$, thus the radial function $F_{n k}(r)$ satisfying the following like Schrödinger equation [21]:

$$
\left[\frac{d^{2}}{d r^{2}}-\frac{k(k+1)}{r^{2}}-M^{2}-E^{2}(M-E)\left(\frac{1}{2} M \omega^{2} r^{2}+\frac{\alpha}{2 M r^{2}}\right)\right] F_{n k}(r)=0
$$

The relativistic energy $E_{n, k}$ and radial upper wave $F_{n k}(r)$ are given by [21]

$$
\frac{M^{2}-E_{n, k}^{2}}{\sqrt{M\left(M+E_{n, k}\right)}}+4 n+2 L+3=0
$$

and

$$
F_{n, k}(r)=C_{n} \exp \left(-\frac{\sqrt{M\left(M+E_{n, k}\right)}}{2} r^{2}\right) r^{L+1} L_{n}^{L+1 / 2}\left(\sqrt{M\left(M+E_{n, k}\right)} r^{2}\right)
$$

where $L_{n}^{L+1 / 2}\left(\sqrt{M\left(M+E_{n, k}\right)} r^{2}\right)$ stands for the associated Laguerre functions. For, the exact pseudospin symmetry which corresponds $\frac{d \Sigma(r)}{d r}=0$, the relativistic energy $E_{n, k}$ and radial lower wave $G_{n k}(r)$ are given by [21]:

$$
\frac{M^{2}-E_{n, k}^{2}}{\sqrt{M\left(M-E_{n, k}\right)}}+4 n+2 \tilde{L}+3=0
$$

and

$$
G_{n, k}(r)=C_{n} \exp \left(-\frac{\sqrt{M\left(M-E_{n, k}\right)}}{2} r^{2}\right) r^{\tilde{L}+1} L_{n}^{\tilde{L}+1 / 2}\left(\sqrt{M\left(M-E_{n, k}\right)} r^{2}\right)
$$

\section{NC relativistic Hamiltonian for (m.a.o.)}

\section{Formalism of Bopp's shift method}

In this section I first highlight in brief the basics of the concepts of the quantum noncommutative quantum mechanics in the framework of relativistic Dirac equation for modified an harmonic oscillator $V_{a o}(\hat{r})$ on based to our works [25,28,29,32,-48]:

a. Ordinary Dirac Hamiltonian operator $\hat{H}\left(p_{i}, x_{i}\right)$ replace by NC Dirac Hamiltonian operator $\hat{H}_{n c-a o}\left(\hat{p}_{i}, \hat{x}_{i}\right)$

b. Ordinary spinor $\Psi(\vec{r})$ replace by new spinor $\hat{\Psi}(\overrightarrow{\vec{r}})$,

c. Ordinary relativistic energy $\mathrm{E}_{\mathrm{nk}}$ replaces by new relativistic 
energy $E_{n c-a o}$ and ordinary product replace by new star product $*$.

Thus, the Dirac equation in ordinary quantum mechanics will change into the Dirac equation in extended quantum mechanics for the (m.a.o.) as follows:

$$
\hat{H}_{n c-a o}\left(\hat{p}_{i}, \hat{x}_{i}\right) * \hat{\Psi}(\overrightarrow{\vec{r}})=E_{n c-a o} \hat{\Psi}(\ddot{\vec{r}})
$$

The Bopp's shift method permutes to reduce the above NC equation to simplest form with usual product and translations applied to in space and phase operators:

$$
H_{n c-a o}\left(\hat{p}_{i}, \hat{x}_{i}\right) \psi(\vec{r})=E_{n c-a o} \psi(\vec{r})
$$

Where the new Hamiltonian operator $H_{n c-a o}\left(\hat{p}_{i}, \hat{x}_{i}\right)$ can be expressed in three general varieties: both NC space and NC phase (NC-3D: RSP), only NC space (NC-3D: RS) and only NC phase (NC: 3D-RP) as, respectively:

$$
H_{n c-a o}\left(\hat{p}_{i}, \hat{x}_{i}\right) \equiv H\left(\hat{p}_{i}=p_{i}-\frac{1}{2} \bar{\theta}_{i j} x_{j} ; \hat{x}_{i}=x_{i}-\frac{1}{2} \theta_{i j} p_{j}\right) \text { for (NC-3D: RSP) }
$$

$$
\begin{aligned}
& H_{n c-a o}\left(\hat{p}_{i}, \hat{x}_{i}\right) \equiv H\left(\hat{p}_{i}=p_{i} ; \hat{x}_{i}=x_{i}-\frac{1}{2} \theta_{i j} p_{j}\right) \text { for }(\mathrm{NC}-3 \mathrm{D}: \mathrm{RS}) \\
& H_{n c-a o}\left(\hat{p}_{i}, \hat{x}_{i}\right) \equiv H\left(\hat{p}_{i}=p_{i}-\frac{1}{2} \bar{\theta}_{i j} ; x_{j}, \hat{x}_{i}=x_{i}\right) \text { for } \quad(\mathrm{NC}-3 \mathrm{D}: \mathrm{RP})
\end{aligned}
$$

In recently work, we are interest with the second variety which present by eq. (29) and by the means of the auxiliary two variables $\hat{x}_{i}=x_{i}-\frac{1}{2} \theta_{i j} p_{j}$ and $\hat{p}_{i}=p_{i}$, the new modified Hamiltonian $H_{n c-a o}\left(\hat{p}_{i}, \hat{x}_{i}\right)$ may be written as follows

$$
H_{n c-a o}\left(\hat{p}_{i}, \hat{x}_{i}\right)=\alpha \hat{\mathrm{P}}+\beta(M+S(\hat{r}))+V_{a o}(\hat{r})
$$

where the modified anharmonic oscillator $V_{a o}(\hat{r})$ is given by:

$$
V_{a o}(\hat{r})=\frac{1}{2} M \omega^{2} \hat{r}^{2}+\frac{\alpha}{2 M \hat{r}^{2}}
$$

The Dirac equation in the presence of above interaction $V(\hat{r})$ can be rewritten according Bopp shift method as follows:

$$
(\alpha \mathrm{P}+\beta(M+S(\hat{r}))) \Psi(r, \theta, \phi)=\left(E_{n c-a o}-V_{a o}(\hat{r})\right) \Psi(r, \theta, \phi)
$$

The radial functions $\left(F_{n k}(r), G_{n k}(r)\right.$ ) are obtained by solving two equations:

$$
\left[\frac{d}{d r}+\frac{k}{r}\right] F_{n k}(r)=\left[M+E_{n c-k b}-\Delta(\hat{r})\right] G_{n k}(r)
$$

$$
\left[\frac{d}{d r}+\frac{k}{r}\right] G_{n k}(r)=\left[M-E_{n c-k b}+\Sigma(\hat{r})\right] G_{n k}(r)
$$

with $\quad \Delta(\hat{r})=V(\hat{r})-S(\hat{r}) \quad$ and $\quad \Sigma(\hat{r})=V(\hat{r})+S(\hat{r})$, eliminating $F_{n k}(r)$ and $G_{n k}(r)$ from Eqs. (34) and (35), we can obtain the following two Schrödinger-like differential equations in (NC-3D: RS) symmetries as follows:

$$
\left[\frac{d^{2}}{d r^{2}}-\frac{k(k+1)}{r^{2}}-\left(M+E_{n c-a o}-\Delta(\hat{r})\right)\left(M-E_{n c-a o}+\Sigma(\hat{r})\right)\right] F_{n k}(r)=0
$$

and

$$
\left[\frac{d^{2}}{d r^{2}}-\frac{k(k-1)}{r^{2}}-\left(M+E_{n c-a o}-\Delta(\hat{r})\right)\left(M-E_{n c-a o}+\Sigma(\hat{r})\right)\right] G_{n k}(r)=0
$$

After straightforward calculations one can obtains the following two terms: $\frac{1}{2} M \omega^{2} \hat{r}^{2}$ and $\frac{\alpha}{2 M \hat{r}^{2}}$ in (NC-3D: RS) as follows:

$$
\begin{aligned}
& \frac{1}{2} M \omega^{2} \hat{r}^{2}=\frac{1}{2} M \omega^{2} r^{2}-\frac{1}{2} M \omega^{2} \overrightarrow{\mathbf{L}} \vec{\Theta} \\
& \frac{\alpha}{2 M \hat{r}^{2}}=\frac{\alpha}{2 M r^{2}}+\frac{\alpha \overrightarrow{\mathbf{L}} \vec{\Theta}}{2 M r^{4}}
\end{aligned}
$$

Which allow us to writing the (m.a.o.) potential $V_{a o}(\hat{r})$ in (NC3D: RS) as follows:

$V_{a o}(\hat{r})=\frac{1}{2} M \omega^{2} r^{2}+\frac{\alpha}{2 M r^{2}}+\left\{\begin{array}{l}\hat{V}_{1 p-a o}(r, \Theta, M, \omega)=\left(\frac{\alpha}{2 M r^{4}}-\frac{1}{2} M \omega^{2}\right) \overrightarrow{\mathbf{L}} \vec{\Theta} \text { for the spin symmetric case } \\ \hat{V}_{2 p-a o}(r, \Theta, M, \omega)=\left(\frac{\alpha}{2 M r^{4}}-\frac{1}{2} M \omega^{2}\right) \tilde{\mathbf{L}} \vec{\Theta} \text { for the p-spin symmetric case }\end{array}\right.$ 
It's clearly that, the first 2-terms represent the ordinary anharmonic oscillator while the rest two parts $\hat{V}_{1 p-a \rho}(r, \Theta, M, \omega)$ and $\hat{V}_{2 p-a o}(r, \Theta, M, \omega)$ are produced by the deformation of space, this allows writing the (m.a.o.) in the NC case as an equation similarly to the usual Dirac equation of the commutative type with a non local potential. Furthermore, using the unit step function (also known as the Heaviside step function or simply the theta function) we can rewrite the modified anharmonic oscillator to the following form:

$$
V_{a o}(\hat{r})=\frac{1}{2} M \omega^{2} r^{2}+\frac{\alpha}{2 M r^{2}}+\theta\left(E_{n c-a o}\right) \hat{V}_{1 p-a o}(r, \Theta, M, \omega)+\theta\left(-E_{n c-a o}\right) \hat{V}_{2 p-a o}(r, \Theta, M, \omega)
$$

Where

$$
\theta(x)=\left\{\begin{array}{lll}
1 & \text { for } & \mathrm{x}>0 \\
0 & \text { for } & \mathrm{x}<0
\end{array}\right.
$$

We generalized the constraint for the pseudospin ( $p$-spin) symmetry $\Delta(r)=V(r)$ and $\Sigma(r)=C_{p s}=$ constants which presented in refs. [18-21] into the new form $\Delta(\hat{r})=V(\hat{r})$ and $\Sigma(\hat{r})=\hat{C}_{p s}=$ constants in (NC-3D: RS) and inserting the potential $V_{a o}(\hat{r})$ in eq. (39) into the two Schrödinger-like differential equations (36) and (37), one obtains:

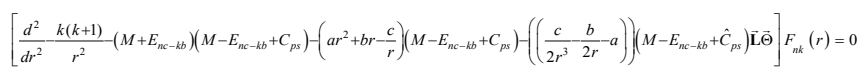



It's clearly that, the additive two parts $\hat{V}_{1 p-a o}(r, \Theta, M, \omega)$ and $\hat{V}_{2 p-a o}(r, \Theta, M, \omega)$ are proportional with infinitesimal parameter $\Theta$, thus we can considered as a perturbations terms.

The Exact Relativistic Spin-Orbital Hamiltonian and the Corresponding Spectrum for (m.a.o.) In (nc: 3drs) Symmetries for $n^{\text {th }}$ Excited States for One-Electron Atoms

The exact relativistic spin-orbital Hamiltonian for (m.a.o.) in (NC: 3D- RS) symmetries for one-electron atoms:

Again, the two perturbative terms $\hat{V}_{1 p-a o}(r, \Theta, M, \omega)$ and $\hat{V}_{2 p-a o}(r, \Theta, M, \omega)$ can be rewritten to the equivalent physical form for (m.a.o.) potential as follows:

$$
\left\{\begin{array}{l}
\hat{V}_{1 p-a o}(r, \Theta, M, \omega)=\Theta\left(\frac{\alpha}{2 M r^{4}}-\frac{1}{2} M \omega^{2}\right) \overrightarrow{\mathbf{L}} \vec{S} \text { for the spin symmetric case } \\
\hat{V}_{2 p-a o}(r, \Theta, M, \omega)=\Theta\left(\frac{\alpha}{2 M r^{4}}-\frac{1}{2} M \omega^{2}\right) \tilde{\overrightarrow{\mathbf{L}}} \overrightarrow{\tilde{S}} \text { for the p-spin symmetric case }
\end{array}\right.
$$

Furthermore, the above perturbative terms $\hat{V}_{1 p-a o}(r, \Theta, M, \omega)$ and $\hat{V}_{2 p-a o}(r, \Theta, M, \omega)$ can be rewritten to the following new equivalent form for (m.a.o.) potential:

$$
\left\{\begin{array}{l}
\hat{V}_{1 p-a o}(r, \Theta, M, \omega)=\frac{1}{2} \Theta\left(\frac{\alpha}{2 M r^{4}}-\frac{1}{2} M \omega^{2}\right)\left(\vec{J}^{2}-\vec{L}^{2}-\vec{S}^{2}\right) \text { for the spin symmetric case } \\
\hat{V}_{2 p-a o}(r, \Theta, M, \omega)=\frac{1}{2} \Theta\left(\frac{\alpha}{2 M r^{4}}-\frac{1}{2} M \omega^{2}\right)\left(\vec{J}^{2}-\vec{L}^{2}-\overrightarrow{\tilde{S}}^{2}\right) \text { for the p-spin symmetric case }
\end{array}\right.
$$

To the best of our knowledge, we just replace the two spinorbital coupling $\vec{S} \vec{L}$ and $\tilde{\overrightarrow{\mathbf{L}}} \overrightarrow{\tilde{S}}$ by the expression $\frac{1}{2}\left(\vec{J}^{2}-\vec{L}^{2}-\vec{S}^{2}\right)$ and $\frac{1}{2}\left(\vec{J}^{2}-\vec{L}^{2}-\overrightarrow{\tilde{S}}^{2}\right)$, in relativistic quantum mechanics.The set ( $H_{n c-k b}\left(\hat{p}_{i}, \hat{x}_{i}\right), \mathrm{J}^{2}, \mathrm{~L}^{2}, \tilde{\mathrm{S}}^{2}$ and $\left.J_{z}\right)$ forms a complete of conserved physics quantities and the spin-orbit quantum number $k(\tilde{k})$ is related to the quantum numbers for spin symmetry $\tilde{l}$ and p-spin symmetry $\tilde{l}$ as follows [18-21]:

$k=\left\{\begin{array}{l}k_{1} \equiv-(l+1) \quad \text { if }-(\mathrm{j}+1 / 2),\left(\mathrm{s}_{1 / 2}, p_{3 / 2}, \text { etc }\right), j=l+\frac{1}{2}, \text { aligned } \operatorname{spin}(\mathrm{k}\langle 0) \\ k_{2} \equiv+l \quad \text { if }\left(j=l+\frac{1}{2}\right),\left(\mathrm{p}_{1 / 2}, d_{3 / 2}, \text { etc }\right), j=l-\frac{1}{2}, \text { unaligned } \operatorname{spin}(\mathrm{k}>0)\end{array}\right.$

and

$\tilde{k}=\left\{\begin{array}{l}\tilde{k}_{1} \equiv-\tilde{l} \quad \text { if }-(\mathrm{j}+1 / 2),\left(\mathrm{s}_{1 / 2}, p_{3 / 2}, \text { etc }\right), j=\tilde{l}-\frac{1}{2}, \text { aligned spin }(\mathrm{k}\langle 0) \\ \left.\tilde{k}_{2} \equiv+(\tilde{l}+1) \quad \text { if }\left(j=\tilde{l}+\frac{1}{2}\right),\left(\mathrm{p}_{1 / 2}, d_{3 / 2}, \text { etc }\right), j=\tilde{l}+\frac{1}{2}, \text { unaligned spin }(\mathrm{k}\rangle 0\right)\end{array}\right.$

With $\tilde{k}(\tilde{k}-1)=\tilde{l}(\tilde{l}+1)$ and $k(k-1)=l(l+1)$, which allows us to form two diagonal $(3 \times 3)$ matrixes $\hat{H}_{s o-a o}\left(k_{1}, k_{2}\right)$ and $\hat{\tilde{H}}_{\text {so-ao }}\left(\tilde{k}_{1}, \tilde{k}_{2}\right)$, for (m.a.o.), respectively, in (NC: 3D-RS) as: 


$$
\begin{aligned}
& \left(\hat{H}_{s o-a o}\right)_{11}\left(k_{1}\right)=k_{1} \Theta\left(\frac{\alpha}{2 M r^{4}}-\frac{1}{2} M \omega^{2}\right) \text { if }-(\mathrm{j}+1 / 2),\left(\mathrm{s}_{1 / 2}, p_{3 / 2}, e t c\right), j=l+\frac{1}{2}, \text { aligned spin }(\mathrm{k}\langle 0) \\
& \left.\left(\hat{H}_{s o-a o}\right)_{22}\left(k_{2}\right)=k_{2} \Theta\left(\frac{\alpha}{2 M r^{4}}-\frac{1}{2} M \omega^{2}\right) \text { if }\left(j=l+\frac{1}{2}\right),\left(\mathrm{p}_{1 / 2}, d_{3 / 2}, \text { etc }\right), j=l-\frac{1}{2}, \text { unaligned spin }(\mathrm{k}\rangle 0\right) \\
& \left(\hat{H}_{s o-a o}\right)_{33}=0
\end{aligned}
$$

and

$$
\begin{aligned}
& \left(\hat{H}_{s o-a o}\right)_{11}\left(\tilde{k}_{1}\right)=\tilde{k}_{1} \Theta\left(\frac{\alpha}{2 M r^{4}}-\frac{1}{2} M \omega^{2}\right) \text { if }-(\mathrm{j}+1 / 2),\left(\mathrm{s}_{1 / 2}, p_{3 / 2}, e t c\right), j=\tilde{l}-\frac{1}{2}, \text { aligned spin }(\mathrm{k}\langle 0) \\
& \left.\left(\hat{H}_{s o-a o}\right)_{22}\left(\tilde{k}_{2}\right)=\tilde{k}_{2} \Theta\left(\frac{\alpha}{2 M r^{4}}-\frac{1}{2} M \omega^{2}\right) \text { if }\left(j=\tilde{l}+\frac{1}{2}\right),\left(\mathrm{p}_{1 / 2}, d_{3 / 2}, e t c\right), j=\tilde{l}+\frac{1}{2}, \text { unaligned spin }(\mathrm{k}\rangle 0\right) \\
& \left(\hat{H}_{s o-a o}\right)_{33}=0
\end{aligned}
$$

The exact relativistic spin-orbital spectrum for (m.a.o.) potential symmetries for $n^{\text {th }}$ excited states for one-electron atoms in (NC: 3D- RSP) symmetries:

In this sub section, we are going to study the modifications to the energy levels $E_{n c-p e r: u}\left(\Theta, k_{1}, E_{n k}, M, \omega\right)$ and $E_{n c-p e r: d}\left(\Theta, k_{2}, E_{n k}, M, \omega\right)$ for $\left(-(\mathrm{j}+1 / 2),\left(\mathrm{s}_{1 / 2}, p_{3 / 2}, e t c\right), j=l+\frac{1}{2}\right.$, aligned spin $\left(\mathrm{k}\langle 0)\right.$ and spin-up) and $\left(\left(j=l+\frac{1}{2}\right),\left(\mathrm{p}_{1 / 2}, d_{3 / 2}, e t c\right), j=l-\frac{1}{2}\right.$, unaligned spin $\left.(\mathrm{k}\rangle 0\right)$ and spin down), respectively, at first order of infinitesimal parameter $\Theta$, for ground state, obtained by applying the standard perturbation theory, using Eqs. (22), (44)and (45) as:

$$
\begin{aligned}
& \int \stackrel{+}{\Psi}_{n k}(r, \theta, \phi)\left[\theta\left(E_{n c-a o}\right) \hat{V}_{1 p-a o}\left(r, \Theta, E_{n k}, M, \omega\right)+\theta\left(-E_{n c-a o}\right) \hat{V}_{2 p-a o}\left(r, \Theta, E_{n k}, M, \omega\right)\right] \Psi_{n k}(r, \theta, \phi) r^{2} d r d \Omega= \\
& =\theta\left(E_{n c-a o}\right) \int F_{n k}^{*}(r) \hat{V}_{1 p-k b}\left(r, \Theta, E_{n k}, M, \omega\right) F_{n k}(r) d r-\theta\left(E_{n c-a o}\right) \int G_{n \hat{k}}^{*}(r) \hat{V}_{2 p-a o}\left(r, \Theta, E_{n k}, M, \omega\right) G_{n \tilde{k}}(r) d r
\end{aligned}
$$

The first parts represent the modifications to the energy levels for the spin symmetric cases $E_{n c-p e r: u}\left(\Theta, k_{1}, E_{n k}, M, \omega\right)$ and $E_{n c-p e r: d}\left(\Theta, k_{2}, E_{n k}, M, \omega\right)$ while the second part represent the modifications to the energy levels $\left(E_{n c-p e r: d}\left(\Theta, \tilde{k}_{1}, E_{0 k}, E_{n k}, M, \omega\right)\right.$, $\left.E_{n c-p e r: u}\left(\Theta, \tilde{k}_{2}, E_{0 k}, E_{n k}, M, \omega\right)\right)$ for the spin spin-symmetry, then we have explicitly:

$$
\begin{aligned}
& E_{n c-p e r: u}\left(\Theta, k_{1}, E_{n k}, M, \omega\right) \equiv \theta\left(E_{n c-k b}\right) k_{1} \Theta \int \stackrel{*}{F_{n k}}(r)\left(\frac{\alpha}{2 M r^{4}}-\frac{1}{2} M \omega^{2}\right) F_{n k}(r) d r \\
& E_{n c-p e r: u}\left(\Theta, k_{2}, E_{n k}, M, \omega\right) \equiv \theta\left(E_{n c-k b}\right) k_{2} \Theta \int^{*} F_{n k}(r)\left(\frac{\alpha}{2 M r^{4}}-\frac{1}{2} M \omega^{2}\right) F_{n k}(r) d r
\end{aligned}
$$

Inserting the radial function $F_{k}(r)$ given by Eq. (23) into the above two Eqs. (51) and (52) to obtain: 


$$
\begin{aligned}
& E_{n c-p e r: u}\left(\Theta, k_{2}, E_{n k}, M, \omega\right) \equiv \theta\left(E_{n c-a o}\right) \Theta k_{1}\left|C_{n}\right|^{2} \int_{0}^{+\infty} \exp \left(-\sqrt{M\left(M+E_{n, k}\right)} r^{2}\right) r^{2 L+2}\left[L_{n}^{L+1 / 2}\left(\sqrt{M\left(M+E_{n, k}\right)} r^{2}\right)\right]^{2}\left(\frac{\alpha}{2 M r^{4}}-\frac{1}{2} M \omega^{2}\right) d r \\
& E_{n c-p e r: u}\left(\Theta, k_{2}, E_{n k}, M, \omega\right) \equiv \theta\left(E_{n c-a o}\right) \Theta k_{2}\left|C_{n}\right|^{2} \int_{0}^{+\infty} \exp \left(-\sqrt{M\left(M+E_{n, k}\right)} r^{2}\right) r^{2 L+2}\left[L_{n}^{L+1 / 2}\left(\sqrt{M\left(M+E_{n, k}\right)} r^{2}\right)\right]^{2}\left(\frac{\alpha}{2 M r^{4}}-\frac{1}{2} M \omega^{2}\right) d r
\end{aligned}
$$

To evaluate the integrations here, we rewriting the above two integrals to the useful forms:

$$
\begin{aligned}
& E_{n c-\text { per: } u}\left(\Theta, k_{2}, E_{n k}, M, \omega\right) \equiv \theta\left(E_{n c-a o}\right) \Theta k_{1}\left|C_{n}\right|^{2} \sum_{\mu=1}^{2} T_{a o}^{\mu}\left(E_{n k}, M, \omega\right) \\
& E_{n c-p e r: u}\left(\Theta, k_{2}, E_{n k}, M, \omega\right) \equiv \theta\left(E_{n c-a o}\right) \Theta k_{2}\left|C_{n}\right|^{2} \sum_{\mu=1}^{2} T_{a o}^{\mu}\left(E_{n k}, M, \omega\right)
\end{aligned}
$$

Where the factors $T_{a o}^{\mu}\left(E_{n k}, M, \omega\right)(\mu=1,2)$ are given by:

$$
\begin{aligned}
& T_{a o}^{1}\left(E_{n k}, M, \omega\right)=\frac{\alpha}{2 M} \int_{0}^{+\infty} \exp \left(-\sqrt{M\left(M+E_{n, k}\right)} r^{2}\right) r^{2 L-2}\left[L_{n}^{L+1 / 2}\left(\sqrt{M\left(M+E_{n, k}\right)} r^{2}\right)\right]^{2} d r \\
& T_{a o}^{2}\left(E_{n k}, M, \omega\right)=-\frac{1}{2} M \omega^{2} \int_{0}^{+\infty} \exp \left(-\sqrt{M\left(M+E_{n, k}\right)} r^{2}\right) r^{2 L+2}\left[L_{n}^{L+1 / 2}\left(\sqrt{M\left(M+E_{n, k}\right)} r^{2}\right)\right]^{2} d r
\end{aligned}
$$

The above two equations, after employing an appropriate coordinate transformation $r^{2}=t$, transforms to the following form:

$$
\begin{aligned}
& T_{a o}^{1}\left(E_{n k}, M, \omega\right)=\frac{\alpha}{4 M} \int_{0}^{+\infty} \exp \left(-\sqrt{M\left(M+E_{n, k}\right)}\right) t\left(t^{L-\frac{1}{2}}\right)-1\left[L_{n}^{L+1 / 2}\left(\sqrt{M\left(M+E_{n, k}\right)} t\right)\right]^{2} d t \\
& T_{a o}^{2}\left(E_{n k}, M, \omega\right)=-\frac{1}{4} M \omega^{2} \int_{0}^{+\infty} \exp \left(-\sqrt{M\left(M+E_{n, k}\right)}\right) t\left(L+\frac{3}{2}\right)-1\left[L_{n}^{L+1 / 2}\left(\sqrt{M\left(M+E_{n, k}\right)} t\right)\right]^{2} d t
\end{aligned}
$$

Now, to obtain the modifications to the energy levels for $n^{\text {th }}$ excited states we apply the following special integration [69]:

$$
\int_{0}^{+\infty} t^{\alpha-1 .} \exp (-\delta t) \Gamma_{m}^{\lambda}(\delta t) \Gamma_{n}^{\beta}(\delta t) d t=\frac{\delta^{-\alpha} \Gamma(n-\alpha+\beta+1) \Gamma(m+\lambda+1)}{m ! n ! \Gamma(1-\alpha+\beta) \Gamma(1+\lambda)}{ }_{3} F_{2}(-m, \alpha, \alpha-\beta ;-n+\alpha, \lambda+1 ; 1)
$$

where ${ }_{3} F_{2}(-m, \alpha, \alpha-\beta ;-n+\alpha, \lambda+1 ; 1)$ obtained from the generalized the hypergeometric function ${ }_{p} F_{q}\left(\alpha_{1}, \ldots, \alpha_{p}, \beta_{1}, \ldots ., \beta_{q}, z\right)$ for $q=2$ and $q=2$ while $\Gamma(x)$ denote to the usual Gamma function. After straightforward calculations, we can obtain the explicitly results: 


$$
\begin{aligned}
& T_{a o}^{1}\left(E_{n k}, M, \omega\right)=\frac{\alpha}{4 M} \frac{\left[M\left(M+E_{n, k}\right)\right]^{-\frac{2 L-1}{4}} \Gamma(n+2) \Gamma(n+L+3 / 2)}{(n !)^{2} \Gamma(2) \Gamma(L+3 / 2)}{ }_{3} F_{2}(-n, L-1 / 2,-1 ; L-n-1 / 2, L+3 / 2 ; 1) \\
& T_{a o}^{2}\left(E_{n k}, M, \omega\right)=-\frac{1}{4} M \omega^{2} \frac{\left[M\left(M+E_{n, k}\right)\right]^{-\frac{2 L+3}{4}} \Gamma(n) \Gamma(n+L+3 / 2)}{(n !)^{2} \Gamma(L+3 / 2)}{ }_{3} F_{2}\left(-n, L+\frac{3}{2}, 1 ; L-n+3 / 2, L+3 / 2 ; 1\right)
\end{aligned}
$$

Hence the exact modifications $E_{n c-p e r: u}\left(\Theta, k_{1}, E_{n k}, M, \omega\right)$ and $E_{n c-p e r: d}\left(\Theta, k_{2}, E_{n k}, M, \omega\right)$ of $E_{n c-p e r: d}\left(\Theta, k_{2}, E_{n k}, M, \omega\right)$ excited states which produced by spin-orbital effect:

$$
\begin{aligned}
& E_{n c-\text { per: } u}\left(\Theta, k_{2}, E_{n k}, M, \omega\right) \equiv \theta\left(E_{n c-a o}\right) \Theta k_{1}\left|C_{n}\right|^{2} T_{a o}\left(E_{n k}, M, \omega\right)(61) \\
& E_{n c-\text { per: }:}\left(\Theta, k_{2}, E_{n k}, M, \omega\right) \equiv \theta\left(E_{n c-a o}\right) \Theta k_{2}\left|C_{n}\right|^{2} T_{a o}\left(E_{n k}, M, \omega\right)
\end{aligned}
$$

Where $T_{a o}\left(E_{n k}, M, \omega\right)$ is the sum of two factors $T_{a o}^{1}\left(E_{n k}, M, \omega\right)$ and $T_{a o}^{2}\left(E_{n k}, M, \omega\right)$.

The exact relativistic magnetic spectrum for (m.a.o.) for $n^{\text {th }}$ excited states for one-electron atoms in (NC: 3D- RS) symmetries:

Having obtained the exact modifications to the relativistic energy levels $E_{n c-p e r: u}\left(\Theta, k_{1}, E_{n k}, M, \omega\right)$ and $E_{n c-p e r: d}\left(\Theta, k_{2}, E_{n k}, M, \omega\right)$ for $n^{\text {th }}$ excited states which produced with relativistic NC spin-orbital Hamiltonian operator, our objective now, we consider another interested physically meaningful phenomena, which also can be produce from the perturbative terms of anharmonic oscillator related to the influence of an external uniform magnetic field, it's sufficient to apply the following two replacements to describing these phenomena:

$$
\begin{aligned}
& \left(\frac{\alpha}{2 M r^{4}}-\frac{1}{2} M \omega^{2}\right)\left\{\begin{array}{l}
\overrightarrow{\mathbf{L}} \vec{\Theta} \rightarrow \rightarrow \chi\left(\frac{\alpha}{2 M r^{4}}-\frac{1}{2} M \omega^{2}\right) \vec{B} \vec{L} \text { for the spin symmetric case } \\
\overrightarrow{\tilde{\mathbf{L}}} \vec{\Theta} \rightarrow \rightarrow \chi\left(\frac{\alpha}{2 M r^{4}}-\frac{1}{2} M \omega^{2}\right) \vec{B} \tilde{\mathbf{L}} \text { for the p-spin symmetric case }
\end{array}\right. \\
& \Theta \rightarrow \chi B
\end{aligned}
$$

here $\chi$ is infinitesimal real proportional's constants, and we choose the magnetic field $\vec{B}=B \vec{k}$ for simplify the calculations, which allow us to introduce the modified new magnetic Hamiltonian $\hat{H}_{m a g-a o}\left(r, E_{n k}, M, \omega, \chi\right)$ on the (NC: 3D-RS), as:

$$
\hat{H}_{\text {mag }-a o}\left(r, E_{n k}, M, \omega, \chi\right)=\chi\left(\frac{\alpha}{2 M r^{4}}-\frac{1}{2} M \omega^{2}\right)\left\{\begin{array}{l}
(\vec{B} \vec{J}-\overrightarrow{\tilde{S}} \vec{B}) \text { for the spin symmetric case } \\
(\vec{B} \vec{J}-\overrightarrow{\tilde{S}} \vec{B}) \text { for the p-spin symmetric case }
\end{array}\right.
$$


where $(-\vec{S} \vec{B}, \overrightarrow{\tilde{S}} \vec{B})$ denotes to the two ordinary and pseudo Hamiltonians of Zeeman effect. To obtain the exact NC magnetic modifications of energy $E_{\text {mag-ao }}\left(\chi, m, E_{n k}, M, \omega\right)$ for (m.a.o.) under spin-symmetry case which produced automatically from the effect of operator $\hat{H}_{\text {mag-ao }}\left(r, E_{n k}, M, \omega, \chi\right)$, we make the following two simultaneously replacements:

$$
k_{1} \rightarrow \mathrm{m} \quad \text { and } \quad \Theta \rightarrow \chi \quad(65)
$$

Then, the relativistic magnetic modification of energy $E_{\text {mag-ao }}\left(\chi, m, E_{n k}, M, \omega\right)$ corresponding ground state on the (NC-3D: RS) symmetries, can be determined from the following relation:

$$
E_{\text {mag-ao }}\left(\chi, m, E_{n k}, M, \omega\right)=\theta\left(E_{n c-a o}\right) \chi m B \Theta\left|C_{n}\right|^{2} T_{a o}\left(E_{n k}, M, \omega\right)(66)
$$

Where $m$ denote to the angular momentum quantum number satisfying the interval, $-l \leq m \leq+l$, which allow us to fixing ( $2 l+1$ ) values for this quantum number.

\section{Themain results of exact modified global spectrum for (m.a.o.) for one-electron atoms under spin-symmetry and p-spin symmetry in (NC: 3D-RS):}

This principal part of the paper is devoted to the presentation of the several results obtained in the previous sections, we resume the $n^{\text {th }}$ excited states eigenenergies $\left(E_{n c-u}\left(\Theta, k_{1}, \chi, n, m, E_{n k}, M, \omega\right), E_{n c-d}\left(\Theta, k_{2}, \chi, n, m, E_{n k}, M, \omega\right)\right)$ of modified Dirac equation corresponding for $\left(-(\mathrm{j}+1 / 2),\left(\mathrm{s}_{1 / 2}, p_{3 / 2}\right.\right.$, etc $), j=l+\frac{1}{2}$, aligned spin $\mathrm{k}\langle 0$ and spin-down $)$ and $\left(j=l+\frac{1}{2},\left(\mathrm{p}_{1 / 2}, d_{3 / 2}\right.\right.$, etc $), j=l-\frac{1}{2}$, un aligned spin $\left.\mathrm{k}\right\rangle 0$ and spin up), respectively, at first order of parameter $\Theta$, for (m.a.o.) potential in (NC: 3D-RS), respectively, on based to the obtained new results(61), (62) and (66), in addition to the original results (22) of energies in commutative space, we obtain the following original results:

$$
E_{n c-u}\left(\Theta, k_{1}, \chi, n, m, E_{n k}, M, \omega\right)=E_{n k_{1}}+\theta\left(E_{n c-a o}\right) \Theta k_{1}\left|C_{n}\right|^{2} T_{a o}\left(E_{n k}, M, \omega\right)+\theta\left(E_{n c-a o}\right) \chi m B \Theta\left|C_{n}\right|^{2} T_{a o}\left(E_{n k}, M, \omega\right)(67)
$$

and

$$
E_{n c-d}\left(\Theta, k_{2}, \chi, n, m, E_{n k}, M, \omega\right)=E_{n k_{2}}+\theta\left(E_{n c-a o}\right) \Theta k_{2}\left|C_{n}\right|^{2} T_{a o}\left(E_{n k}, M, \omega\right)+\theta\left(E_{n c-a o}\right) \chi m B \Theta\left|C_{n}\right|^{2} T_{a o}\left(E_{n k}, M, \omega\right)(68)
$$

As it is montionated in [18], in view of exact spin symmetry in commutative space $\left(E_{\text {kk }} \rightarrow-E_{k}, V(r) \rightarrow-V(r), k \rightarrow k+1\right.$ and $F_{n k}(r) \rightarrow G_{n \tilde{k}}(r)$ ), we need to generalize the above translations to the case of NC three dimensional spaces, then the negative values $E_{n c-u}\left(\Theta, \tilde{k}_{1}, \chi, m, n, E_{n k}, M, \omega\right)$ and $E_{n c-d}\left(\Theta, \tilde{k}_{2}, \chi, m, n, E_{n k}, M, \omega\right)$ are obtained as:

$$
\begin{aligned}
& E_{n c-u}\left(\Theta, k_{1}, \chi, m, n, E_{n k}, M, \omega\right) \rightarrow E_{n c-u}\left(\Theta, \tilde{k}_{1}, \chi, m, n, E_{n k}, M, \omega\right) \equiv-E_{n c-u}\left(\Theta, k_{1}, \chi, m, n, E_{n k}, M, \omega\right) \\
& E_{n c-d}\left(\Theta, k_{2}, \chi, n, m, E_{n k}, M, \omega\right) \rightarrow E_{n c-d}\left(\Theta, \tilde{k}_{2}, \chi, m, n, E_{n k}, M, \omega\right) \equiv-E_{n c-d}\left(\Theta, k_{2}, \chi, m, n, E_{n k}, M, \omega\right) \\
& V(\hat{r}) \rightarrow-V(\hat{r}) \\
& \tilde{k}_{1} \rightarrow k_{1}+1 \quad \text { and } \quad \tilde{k}_{2} \rightarrow k_{2}+1
\end{aligned}
$$


It's clearly, that the obtained eigenvalues of energies are real is Hermitian; consequently, the modified quantum Hamiltonian operator $\hat{H}_{n c-a o}\left(\hat{p}_{i}, \hat{x}_{i}\right)$ is Hermitian and may be expressed as follows:

$$
\hat{H}_{n c-a o}\left(\hat{p}_{i}, \hat{x}_{i}\right)=\hat{H}_{c o m-a o}\left(p_{i}, x_{i}\right)+\left\{\begin{array}{l}
\Theta\left(\frac{\alpha}{2 M r^{4}}-\frac{1}{2} M \omega^{2}\right) \vec{S} \vec{L}+\chi\left(\frac{\alpha}{2 M r^{4}}-\frac{1}{2} M \omega^{2}\right)(\vec{B} \vec{J}-\overrightarrow{\tilde{S}} \vec{B}) \text { for the spin symmetric case } \\
\Theta\left(\frac{\alpha}{2 M r^{4}}-\frac{1}{2} M \omega^{2}\right) \overrightarrow{\tilde{S}} \vec{L}+\chi\left(\frac{\alpha}{2 M r^{4}}-\frac{1}{2} M \omega^{2}\right)(\vec{B} \vec{J}-\overrightarrow{\tilde{S}} \vec{B}) \text { for the p-spin symmetric case }
\end{array}\right.
$$

Where $\hat{H}_{\text {com-ao }}\left(p_{i}, x_{i}\right)$ is given by:

$$
\hat{H}_{c o m-a o}\left(p_{i}, x_{i}\right)=\alpha \mathrm{P}+\beta(M+S(r))+\frac{1}{2} M \omega^{2} r^{2}+\frac{\alpha}{2 M r^{2}}
$$

Denote to the ordinary Hamiltonian operator in the commutative space. In this way, one can obtain the complete energy spectra for (m.a.o.) potential in (NC: 3D-RS) symmetries. Know the following accompanying constraint relations:

a-The two quantum numbers $(\tilde{m}, m)$ satisfied the two intervals: $-\tilde{l} \leq \tilde{m} \leq+\tilde{l}$ and $-l \leq m \leq+l$, thus we have $2 \tilde{l}+1$ and $2 l+1$ values for these quantum numbers,

b-We have also two values for p-spin symmetry $j=\tilde{l}+\frac{1}{2}$ and $j=\tilde{l}-\frac{1}{2}$ and two values for spin symmetry $j=l+\frac{1}{2}$ and $j=l-\frac{1}{2}$.

Allow us to deduce the important original results: every state in usually three dimensional space will be replace by $4(2 \tilde{l}+1)$ and $4(2 l+1)$ sub-states under p-spin symmetry and spin symmetry, which allow us to fixing the degenerated states to the $4 \sum^{n-1}(2 l+1) \equiv 4 n^{2}$ values in (NC: $\left.3 \mathrm{D}-\mathrm{RS}\right)$ symmetries. It is easy to see that the obtained originally results reduce to the ordinary results described on quantum mechanics when the noncommutativity of space disappears $(\Theta, \chi) \rightarrow(0,0)$, equations (67), (68) and (69) reduces to (22) and (24) and one recovers the standard textbook results. Finally one concludes; our obtained results are sufficiently accurate for practical purposes. These results are in agreement with the ones obtained previously $[38,47]$.

\section{The Important Concluding Remarks}

Let us summarize our results as follows:

The solution procedure presented in this paper is based on the both of Bopp's shift method and standard perturbation theory, we investigate the bound state energies of $n^{\text {th }}$ excited states for (m.a.o.) described on (NC: 3D-RS).
It is found that the energy eigenvalues depend on the dimensionality of the problem and non-relativistic atomic quantum numbers $(j=\tilde{l} \pm 1 / 2, j=l \pm 1 / 2, \tilde{s}= \pm 1 / 2, l, \tilde{l})$ and the two angular momentum quantum numbers $(m, \tilde{m})$ in addition to the infinitesimal parameters $(\Theta, \chi)$.

We have also constructing the corresponding NC Hermitian Hamiltonian operator $\hat{H}_{n c-a o}\left(\hat{p}_{i}, \hat{x}_{i}\right)$ which presented by eq. (70).

The energy eigenvalues are in good agreement with the results previously. Finally, we point out that these exact results (67), (68) and (69) obtained for this new proposed form of the modified potential (39) may have some interesting applications in the study of different quantum mechanical systems, nuclear physics, atomic and molecular physics.

\section{Acknowledgement}

This work was supported with search laboratory of: Physics and Material Chemistry, in Physics department, Sciences facultyUniversity of M'sila, Algeria.

\section{References}

1. MK Bahar, F Yasuk (2013) Fermionic Particles with PositionDependent Mass in the Presence of Inversely Quadratic Yukawa Potential and Tensor Interaction. Pramana Journal of Physics 80(2): 187-197.

2. M Hamzavi, AA Rajabi, H Hassanabi (2010) Exact Pseudospin Symmetry Solution of the Dirac Equation for Spatially-Dependent Mass Coulomb Potential Including Coulomb-Like Tensor Interaction via Asymptotic Iteration Method. Physics Letters A 374(42): 43034307.

3. H Akcay, C Tezcan (2010) Exact Solutions of the Dirac Equation with Harmonic Oscillator Potential Including a Coulomb-Like Tensor Potential. International Journal of Modern Physics C 20(6): 931-940.

4. H Akcay (2009) Dirac Equation with Scalar and Vector Quad-ratic 
Potentials and Coulomb-Like Tensor Potential. Physics Letters A 373(6): 616-620.

5. E Maghsoodi, H Hassanabadi, S Zarrinkamar (2013) Exact Solutions of the Dirac Equation with Poschl-Teller Double-Ring Shaped Coulomb Potential via the Nikiforov-Uvarov Method. Chinese Physics B 22(3): 030302-1-030302-5.

6. C Berkdemir, YF Cheng (2009) On the Exact Solutions of the Dirac Equation with a Novel Angle-Dependent Potential. Physica Scripta 79(3): 035003-035010.

7. Benedict Iserom Ita, Alexander Immaanyikwa Ikeuba (2013) Solutions of the Dirac Equation with Gravitational plus Exponential Potential. Applied Mathematics 4: 1-6

8. Jean Mawhin, André Ronveaux (2010) Schrödinger and Dirac equations for the hydrogen atom and Laguerre polynomials. Arch Hist Exact Sci 64(4): 429-460.

9. B Biswas, S Debnath (2016) Bound States of the Dirac-KratzerFues Problem with Spin and Pseudo-Spin Symmetry via Laplace Transform Approach. Bulg J Phys 43: 89-99.

10. F Pakdel, AA Rajabi (2016) Investigation of the nuclear system using the D-dimensional wave equation. Chinese Journal of Physics 54(3): 385-390.

11. KJ Oyewumi, CO Akoshile (2010) Bound-state solutions of the DiracRosen-Morse potential with spin and pseudospin symmetry. Eur Phys J A 45: 311-318.

12. H Hassanabadi, E Maghsoodi, S Zarrinkamar (2012) Relativistic symmetry of the Dirac equation and Tietz potential. Eur Phys J plus 123: 31.

13. MR Setare, S Haidari (2010) Spin symmetry of the Dirac equation with the Yukawa potential. Phys Scr 81(6): 065201.

14. A Soylu, O Bayrak, I Boztosun (2007) An approximate solution of the Dirac-Hulthen problem with pseudospin and spin symmetry for any k state. J Math Phys 48: 082302.

15. AA Onate, JO Ojonubah (2015) Relativistic and nonrelativistic solutions of the generalized Pöschl-Teller and hyperbolical potentials with some thermodynamic properties. Int J Mod Phys E 24: 1550020

16. SM Ikhdair, R Sever (2010) Approximate Analytical solutions of the generalized woods-Saxon potentials including the spin-orbit coupling term and spin symmetry. Cent Eur J Phys 8(4): 652-666.

17. SM Ikhdair, R Sever (2007) Approximate Eigenvalue and Eigenfunctions Solutions for the Generalized Hulthén Potential with any Angular Momentum. J Math Chem 42(3): 461-471.

18. M Eshghia, SM Ikhdair (2014) Relativistic effect of pseudospin symmetry and tensor coupling on the Mie-type potential via Laplace transformation method. Chin Phys B 23(12): 120304.

19. Sameer M Ikhdair, Majid Hamzavi (2012) Approximate Relativistic Bound State Solutions of the Tietz-Hua Rotating Oscillator for Any к-State. Few-Body Syst 53(3): 473-486.

20. M Hamzavi, AA Rajabi (2011) Solution of Dirac Equation with Killingbeck Potential by Using Wave Function Ansatz Method under Spin Symmetry Limit Commun. Theor Phys 55(1): 35-37.

21. H Goudarzi, M Sohbati, S Zarrin (2011) Solution of Dirac equation with spin and pseudospin symmetry for an anharmonic oscillator. Journal Of Mathematical Physics 52(1): 013506
22. H Snyder (1947) Quantized Space Time. Phys Rev 71: 38-41.

23. Abdelmadjid Maireche (2014) A Study of Schrödinger Equation with Inverse Sextic Potential in 2-dimensional Non-commutative Space. Afr Rev Phys 9(0025): 185-193.

24. Abdelmadjid Maireche (2014) Spectrum of Schrödinger Equation with HLC Potential in Non-Commutative Two-dimensional Real Space. The African Rev Phys 9(0060): 479-483.

25. Abdelmadjid Maireche (2015) Deformed Bound States for Central Fraction Power Potential: Non Relativistic Schrödinger Equation. Afr Rev Phys 10(0014): 97-103.

26. Abdelmadjid Maireche (2015) Spectrum of Hydrogen Atom Ground State Counting Quadratic Term in Schrödinger Equation. The African Rev Phys 10: 177-183.

27. Abdelmadjid Maireche (2015) Atomic Spectrum for Schrödinger Equation with Rational Spherical Type Potential in Non-commutative Space and Phase. The African Review of Physics 10 (0046): 373-381.

28. Abdelmadjid Maireche (2016) A Complete Analytical Solution of the Mie-Type Potentials in Non-commutative 3-Dimensional Spaces and Phases Symmetries. Afr Rev Phys 11: 111-117.

29. Abdelmadjid Maireche (2016) New Exact Energy Eigen-values for (MIQYH) and (MIQHM) Central Potentials: Non-relativistic Solutions. Afr Rev Phys 11(0023): 175-185.

30. Abdelmadjid Maireche (2015) Nonrelativistic Atomic Spectrum for Companied Harmonic Oscillator Potential and its Inverse in both NC-2D: RSP. International Letters of Chemistry. Physics and Astronomy 56: 1-9.

31. Abdelmadjid Maireche (2015) New Exact Solution of the Bound States for the Potential Family $V(r)=A / r^{2}-B / r+C r k(k=0,-1,-2)$ in both Noncommutative Three Dimensional Spaces and Phases: Non Relativistic Quantum Mechanics. International Letters of Chemistry, Physics and Astronomy 58: 164-176.

32. Abdelmadjid Maireche (2015) A New Approach to the Non Relativistic Schrödinger equation for an Energy-Depended Potential $\mathrm{V}(\mathrm{r}, \mathrm{En}, \mathrm{l})=\mathrm{V} 0(1+\boldsymbol{\eta} \mathrm{En}, \mathrm{l}) \mathrm{r}^{2}$ in Both Noncommutative three Dimensional spaces and phases. International Letters of Chemistry, Physics and Astronomy 60: 11-19.

33. Abdelmadjid Maireche (2015) A New Study to the Schrödinger Equation for Modified Potential V(r) $=\mathrm{ar}^{2}+\mathrm{br}^{-4}+\mathrm{cr}^{-6}$ in Nonrelativistic Three Dimensional Real Spaces and Phases. International Letters of Chemistry, Physics and Astronomy 61: 38-48.

34. Abdelmadjid Maireche (2016) A New Nonrelativistic Investigation for the Lowest Excitations States of Interactions in One-Electron Atoms, Muonic, Hadronic and Rydberg Atoms with Modified Inverse Power Potential. International Frontier Science Letters 9: 33-46.

35. Abdelmadjid Maireche (2015) Deformed Quantum Energy Spectra with Mixed Harmonic Potential for Nonrelativistic Schrödinger equation. J Nano- Electron Phys 7(2): 02003-1-02003-6.

36. Abdelmadjid Maireche (2015) A Recent Study of Quantum Atomic Spectrum of the Lowest Excitations for Schrödinger Equation with Typical Rational Spherical Potential at Planck's and Nanoscales. J Nano Electron Phys 7(3): 3047-3051.

37. Abdelmadjid Maireche (2015) Quantum Hamiltonian and Spectrum of Schrödinger Equation with companied Harmonic Oscillator Potential and it's Inverse in three Dimensional Noncommutative Real Space and Phase. J Nano Electron Phys 7(4): 04021-4021-7. 
38. Abdelmadjid Maireche (2016) New Relativistic Atomic Mass Spectra of Quark ( $\mathrm{u}, \mathrm{d}$ and $\mathrm{s}$ ) for Extended Modified Cornell Potential in Nano and Plank's Scales. J Nano Electron Phys 8(1): 01020.

39. Abdelmadjid Maireche (2016) The Nonrelativistic Ground State Energy Spectra of Potential Counting Coulomb and Quadratic Terms in Non-commutative Two Dimensional Real Spaces and Phases. J Nano Electron Phys 8(1): 01021.

40. Abdelmadjid Maireche (2016) New Theoretical Study of Quantum Atomic Energy Spectra for Lowest Excited States of Central (PIHOIQ) Potential in Noncommutative Spaces and Phases Symmetries at Plan's and Nanoscales. J Nano Electron Phys 8(2): 02027-1-0202710

41. Abdelmadjid Maireche (2016) A New Nonrelativistic Atomic Energy Spectrum of Energy Dependent Potential for Heavy Quarkouniom in Noncommutative Spaces and Phases Symmetries. J Nano Electron Phys 8(2): 02046-1-02046-6.

42. Abdelmadjid Maireche, Djenaoui Imane (2016) A New Nonrelativistic Investigation for Spectra of Heavy Quarkonia with Modified Cornell Potential: Noncommutative Three Dimensional Space and Phase Space Solutions. J Nano Electron Phys 8(3): 03024.

43. Abdelmadjid Maireche (2016) New Bound State Energies for Spherical Quantum Dots in Presence of a Confining Potential Model at Nano and Plank's Scales. Nano World J 1(4): 120-127.

44. Abdelmadjid Maireche (2015) New exact bound states solutions for (CFPS) potential in the case of Non-commutative three dimensional non relativistic quantum mechanics. Med J Model Simul 04: 060072.

45. Abdelmadjid Maireche (2014) Quantum Schrödinger equation with Octic potential in non-commutative two-dimensional complex space. Life Sci J 11(6): 353-359.

46. Abdelmadjid Maireche (2015) New Quantum atomic spectrum of Schrödinger equation with pseudo harmonic potential in both noncommutative three dimensional spaces and phases. Lat Am J Phys Educ 09: 1301-1-1301-8.

47. Abdelmadjid Maireche (2016) A New Relativistic Study for Interactions in One-electron atoms (Spin $1 / 2$ Particles) with Modified Mie-type Potential. J Nano Electron Phys 8(4): 04027-1-04027-9.

48. Abdelmadjid Maireche (2016) A new nonrelativistic investigation for interactions in one-electron atoms with modified inverse -square potential: noncommutative two and three dimensional space phase solutions at Planck's and nano-scales. J Nanomed Res 4(3): 0009000105 .

49. Anselme F Dossa, Gabriel YH Avossevou (2013) Noncommutative Phase Space and the Two Dimensional Quantum Dipole in Background Electric and Magnetic Fields. Journal of Modern Physics 4(10): 1400-1411.

50. Jumakari-Mamat, Sayipjamal Dulat, Hekim Mamatabdulla (2016) Landau-like Atomic Problem on a Non-commutative Phase Space. Int J Theor Phys 55: 2913-2918.

51. Yongjun Xiao, Zhengwen Long, Shaohong Cai (2011) Klein-Gordon Oscillator in Noncommutative Phase Space Under a Uniform Magnetic Field. Int J Theor Phys 50: 3105-3111.
52. Abdelmalek Boumali, Hassan Hassanabadi (2013) The thermal properties of a two-dimensional Dirac oscillator under an external magnetic field. Eur Phys J Plus 128: 124.

53. S Dulat, Kang Li (2008) The Aharonov-Casher effect for spin-1 particles in non-commutative quantum mechanics. Eur Phys J C 54(2): 333-337.

54. Curtright T, Fairlie D, Zachos CK (1998) Features of time independent Wigner functions. Phys Rev D 58: 025002.

55. Gamboa J, Loewe M, Rojas JC (2001) Noncommutative quantum mechanics. Phys Rev D 64: 067901.

56. Mezincescu L (2000) Star operation in quantum mechanics. Cornell University Library, USA.

57. Uan Yi, LI Kang, Wang Jian-Hua, chen chi-yi (2010) Spin-1/2 relativistic particle in a magnetic field in NC phase space. Chinese Physics C 34(5): 543.

58. Shaohong Cai, Tao Jing, Guangjie Guo, Rukun Zhang (2010) Dirac Oscillator in Noncommutative Phase Space. International Journal of Theoretical Physics 49(8): 1699-1705.

59. Joohan Lee (2005) Star Products and the Landau Problem. Journal of the Korean Physical Society 47(4): 571-576.

60. Yang Zu-Hua, Chao Yun Long, Shuei Jie Qin, Zheng-Wen Long (2010) DKP Oscillator with spin-0 in Three dimensional Noncommutaive Phase-Space. Int J Theor Phys 49: 644-657.

61. Jumakari-Mamat, Sayipjamal Dulat, Hekim Mamatabdulla (2016) Landau-like Atomic Proplem on a Non-commutative Phase Space. Int J Theor Phys 55(6): 2913-2918.

62. Behrouz Mirza, Rasoul Narimani, Somayeh Zare (2011) Relativistic Oscillators in a Noncommutative space in a Magnetic field. Commun Theor Phys 55: 405-409.

63. Yongjun Xia, Zhengwen Long, Shaohong Cai (2011) Klein-Gordon Oscillator in Noncommutative Phase Space Under a Uniform Magnetic Field. Int J Theor Phys 50: 3105-3111.

64. Al Jamel (2011) Heavy quarkonia with Cornell potential on noncommutative space. Journal of Theoretical and Applied Physics 5(1): 21-24.

65. H Hassanabadi1, F Hoseini, S Zarrinkamar (2015) A generalized interaction in noncommutative space: Both relativistic and nonrelativistic fields. Eur Phys J Plus 130: 200.

66. Won sang, Chung (2015) Two Dimensional Non-commutative Space and Rydberg Atom Model. Int J Theor Phys 54(6): 1840-1849.

67. P Polychronakos, VP Nair (2001) Quantum mechanics on the noncommutative plane and sphere. Physics Letters B 505: 267-274.

68. AEF Djemaï, H Smail (2004) On Quantum Mechanics on Noncommutative Quantum Phase Space. Commun. Theor Phys 41(6): 837-844.

69. M Abramowitz, A Stegun (1965) Handbook of Mathematical Functions with Formulas, Graphs and Mathematical Tables, Dover Publications, New York, USA. 